\title{
Creation of a system for automatic monitoring of electromagnetic effects on biological and technical systems
}

\author{
Evgeniy Grabchak ${ }^{1}$, Vladimir Grigoriev², and Evgeniy Loginov ${ }^{3, *}$ \\ ${ }^{1}$ Ministry of Energy of Russia, 107996, 42 Schepkina, Moscow, Russian Federation \\ ${ }^{2}$ MGIMO (U) Ministry of Foreign Affairs of Russia, 119454, 76 Vernadsky Ave., Moscow, Russian \\ Federation \\ ${ }^{3}$ Situational Analytical Center of the Ministry of Energy of Russia, 107996, Moscow, 42 Schepkina, \\ Russian Federation
}

\begin{abstract}
Using a digital "twin" of a convergent biological and technical supersystem of a certain region to develop measures to ensure environmental safety is because of the need to analyze and predict processes and the level of electromagnetic pollution of the environment because of geomagnetic storms in interaction with the operation of electric power facilities. The article proposes modeling a digital "twin" using indicators of the taxonomy of the identified functional relationships about the influence of natural and man-made electromagnetic fields. The study aims to develop fresh approaches to the development of methods for analyzing the influence of electromagnetic factors on people, animals, plants and technical systems using general and special technologies for recording the levels of pulsed electromagnetic effects. The authors use methods of management theory, expert decision-making methods, principles and approaches of international standards and recommendations in management, ecology and informatics. The article proposes an analysis and identification of the reactions of the digital "twin" of a convergent biological and technical supersystem of a certain region to different categories of signals (information) about the effect of a complex of electromagnetic pollutants, with the determination of indicators of environmental safety and critical electromagnetic effects. The new technology provides for the study of the amplitude of electromagnetic pulses in a wide frequency range, their interpretation, analysis and development of recommendations for maintaining the required modes of environmental monitoring. The necessity of creating new cyber-physical environmental monitoring systems based on the operation of the automatic monitoring system of electromagnetic influences based on noise-resistant sensors for registering the levels of pulsed electromagnetic influences is substantiated.
\end{abstract}

* Corresponding author: loginovel@mail.ru 


\section{Introduction}

Digital technologies open up new, previously unavailable possibilities for monitoring and modeling the ecological state of the elements of a convergent biological and technical supersystem of a certain region $[1,2,3,4]$. It becomes possible to withstand a wide range of natural and technical threats to electromagnetic pollution of the environment because of geomagnetic storms with the operation of electric power facilities [5, 6].

The source of electromagnetic fields and various radio emissions is a sharp change in the geomagnetic field, causing an increase in the electromagnetic activity of electrical networks and other energy objects.

Perturbations of the Earth's magnetic field during periods of geomagnetic storms generate the effect of electromagnetic fields. The problem of countering the threats of electromagnetic pollution of the environment is the lack of a system for automatic monitoring of electromagnetic influences based on noise-resistant sensors for registering the levels of pulsed electromagnetic influences.

\section{Methods}

The study characterizes geomagnetic storms as a threat of electromagnetic effects, substantiates the need to use a digital "twin" of a convergent biological and technical supersystem of a certain region to analyze and predict electromagnetic pollution of the environment, describes the procedures for identifiable reactions of the digital "twin" of a convergent biological and technical supersystem to different categories of information messages from sensors of the system of automatic monitoring of electromagnetic influences, integrated with the modules for the formation of computational models. The authors analyze the indicators of the observed electromagnetic influences, considering the need to respond to influencing factors, and plan measures for the development of a system for automatic monitoring of electromagnetic influences to maintain environmental safety.

\section{Results}

Geomagnetic storms cause a variety of emergencies and crisis situations: deterioration of the state of biological objects, functional disturbances and failures of electrical equipment in wire communication systems, in electric power systems, signaling and communication systems, increased electromagnetic impact on the environment [7].

The main negative factor of the impact of geomagnetic storms is the generation of electromagnetic fields. The scale of the impact predicts emergencies in many Russian territories, which carry potential risks of the influence of electromagnetic factors on people, animals, plants and technical systems. Such a negative impact of geomagnetic disturbances is especially important for the northern (arctic) regions of our country.

The continued expansion of high-voltage power grids, increased loads and the transition to low-impedance transmission lines with higher voltage lead to the likelihood of failures during cosmic electromagnetic phenomena. The expansion of power lines in recent decades has made the power grid complex the equivalent of a large antenna that is electromagnetically coupled to disturbances in the Earth's magnetosphere.

The simplest way to identify threats to electromagnetic pollution in the environment is a natural experiment [8]. The peculiarities of the operation of many natural and technical objects exclude or complicate the conduct of field experiments: it is impossible to create risks for the operation of life support systems and the health of biological objects [9]. 
If it is impossible to conduct a natural experiment, then a digital experiment is possible based on the formation of a digital "twin" of a convergent biological and technical supersystem of a certain region [10]. To do this, we need to analyze and identify the reactions of the digital "twin" of the convergent biological and technical supersystem to different categories of signals (information) about the manifestation of the effect of a complex of electromagnetic pollutants on people, animals, plants and technical systems.

Using a digital "twin" can form a library of situations of manifestation of the influence of electromagnetic pollutants on people, animals, plants and technical systems. The library of situations localized within the framework of the structural and functional variability of the behavior of the model of the digital "twin" of the convergent biological and technical supersystem, determines the indicators of environmental safety and supports various types of management actions in the sustainable behavior of the convergent biological and technical supersystem under the influence of natural and man-made electromagnetic fields.

Let us consider the organization of information mechanisms for analyzing the effect of a set of electromagnetic factors on people, animals, plants and technical systems using general and special technologies for analyzing and interpreting technical information coming from sensors for registering the levels of pulsed electromagnetic influences.

Descriptions of databases on the amplitudes of electromagnetic pulses available for analysis are superimposed on the identified reactions of the digital "twin" of the convergent biological and technical supersystem of a certain region, considering the factors affecting the state of people, animals, plants and technical systems. The descriptions consider the conditions of the minimum starting level of electromagnetic fields, slightly exceeding the level of the background electromagnetic environment. Means for detecting and generating signals characterizing the amplitudes of electromagnetic pulses in a wide frequency range are used to study electromagnetic pollution of the environment with a focus on expanding the general capabilities digital control of objects and system-wide mechanisms for ensuring environmental safety.

Functional and technological connections between the identified parameters of electromagnetic pulses during the developing digitalization of sensors for recording the levels of pulsed electromagnetic effects allow remotely calculating the state of people, animals, plants and technical systems and integrating with the results of monitoring control signals based on the model of a digital "twin" of convergent biological and technical supersystems. The calculated parameters within the framework of modeling to maintain the required modes of environmental monitoring based on the structural and functional variability of the behavior of the model of the digital "twin" of the convergent biological and technical supersystem in new cyber-physical systems of environmental monitoring must be compared with the real parameters got from the interfaces of the automatic monitoring system of electromagnetic influences.

\section{Discussion}

The identified reactions of the digital "twin" of the convergent biological and technical supersystem are typified into different categories of information messages (from sensors of the automatic monitoring system of electromagnetic influences), integrated with the modules for generating computational models. The description of each type of identified reactions of the digital "twin" to different categories of structured data of electromagnetic influences contains a specific set of attributes.

The identified reactions of the digital "twin" of a convergent biological and technical supersystem to different categories of structured signals about electromagnetic influences are represented by their descriptions [11]. Descriptions can be grouped into information 
blocks with output to conclusions about the modalities of electromagnetic pollution of the environment [12].

A set of information blocks about electromagnetic influences and identifiable reactions of the digital "twin" make up a distributed metadata pool. Computational information processing interfaces work with a pool of data using the modalities of the influence of natural and man-made electromagnetic fields in various conditions.

The complex of digital technologies of the system for automatic monitoring of electromagnetic influences can superimpose signals received from noise-immune sensors for registering the levels of pulsed electromagnetic influences on a library of situations and identify critical signals.

Based on the results of registration of the levels of pulsed electromagnetic influences (analysis and interpretation), it is possible to identify the manifestations of electromagnetic fields and various radio emissions, with the identification of signals leading to emergencies.

Dangerous situations because of critical levels of pulsed electromagnetic influences are found by detecting and generating signals characterizing the amplitudes of electromagnetic pulses in a wide frequency range, and processing registration data and interpreting the amplitude of electromagnetic pulses with technological tests and monitoring the behavior of the observed array of electrical networks.

Analysis of the totality of electromagnetic factors, electric field strength and magnetic field strength or magnetic field induction as markers of emergencies reveals the biological impact of electromagnetic fields on the human body. The expansion of the general capabilities of digital control of the object and system-wide mechanisms for ensuring environmental safety as a set of identified relationships occurs through the analysis of the effect of the electromagnetic field created by power lines. For example, a field strength of $1000 \mathrm{~V} / \mathrm{m}$ creates a real danger to people, flora and fauna.

The data on the modalities of electromagnetic pollution of the environment in the action's framework of high-energy electromagnetic fields related to the microwave range of cellular communications are analyzed.

Based on multi-format data integration, they are linked according to complex optimization parameters in the space of functional services of modeling tools for a convergent biological and technical supersystem of a certain region to maintain the required modes of environmental monitoring in new cyber physical environmental monitoring systems.

The analysis of the totality of manifestations of electromagnetic fields and various radio emissions is carried out considering the need to ensure high-quality radio communication and data transmission without distortion in a certain frequency range. The results of the analysis will help in predicting potential emergencies, developing recommendations for the operation of environmental monitoring systems, and identifying actual situations.

The analysis of indicators of electromagnetic influences, which underlie potential crisis situations, considering the need to respond to influencing factors, is carried out. The identified indicators of the observed electromagnetic effects are combined into a certain value that characterizes the efficiency of the vital functions of objects of a convergent biological and technical supersystem of a certain region. This value is compared with the reference values from the situation library, which helps to identify the actual situations and draw attention to the identified source of electromagnetic disturbances.

We can apply similar approaches to solve other environmental problems. For example, to identify electromagnetic pollution of the environment by identifying from the total mass of impacts from power lines and various power plants. Such installations form electromagnetic fields of industrial frequency $(50 \mathrm{~Hz})$, which are hundreds of times higher than the average levels of natural fields. 
The monitoring of the reliability of the information coming from the sensors for registering the levels of pulsed electromagnetic effects can be carried out based on modeling the processes of electromagnetic pollution of the environment within the framework of the "digital twin".

The complex of digital "twin" models reduces the likelihood of non-standard situations and eliminates the factors that determine the adoption of managerial decisions leading to erroneous technological modes that negatively affect the stability of power supply and cellular communications [13].

\section{Conclusion}

It is proposed to create new cyber-physical environmental monitoring systems based on automatic monitoring of electromagnetic effects to analyze the totality of indicators of observed electromagnetic effects. Electromagnetic interference can cause emergencies. The combination of the identified indicators of electromagnetic influences is possible in a certain value that characterizes the life safety of elements (objects) of a convergent biological and technical supersystem of a certain region, in the conditions of a combination of manifestations of electromagnetic fields and various radio emissions [14]. This value is compared with the reference values from the situation library, which allows us to identify the situation.

It is necessary to solve the following tasks to maintain environmental safety with the development of new cyber-physical systems for environmental monitoring:

- to develop a digital "twin" of a convergent biological and technical supersystem of a certain region;

- to develop a system for automatic monitoring of electromagnetic influences based on noise-resistant sensors for registering the levels of pulsed electromagnetic influences;

- to develop a subsystem for determining the reliability of information coming from sensors for registering the levels of pulsed electromagnetic influences;

- to develop services for calculating the parameters of electromagnetic pollution of the environment in the optimal computational mode because of the operation of the digital "twin" of the convergent biological and technical supersystem of a certain region with the operation of the automatic monitoring system of electromagnetic influences;

- to develop a subsystem for supporting management decisions while ensuring environmental safety.

The system of the digital "twin" of a convergent biological and technical supersystem of a certain region should include a database with a set of standard situations and a set of commands that allow for simplified development of recommendations for ensuring environmental safety [15].

The new technology provides for the study of the amplitude of electromagnetic pulses in a wide frequency range, their interpretation, analysis and maintenance of the required environmental safety regimes based on the structural and functional variability of the behavior of the digital "twin" model of a convergent biological and technical supersystem of a certain region in new cyber physical environmental monitoring systems based on noise-resistant sensors for registering the levels of pulsed electromagnetic influences.

\section{References}

1. L. Bodewein, K. Schmiedchen, D. Dechent, D. Stunder, D. Graefrath, L. Winter, T. Kraus, S. Driessen, Environmental Research, 171 (2019)

2. C. Yu, R.-Y. Peng, Military Medical Research 4, 1 (2017) 
3. J. Juutilainen, A. Höytö, T. Kumlin, J. Naarala, Bioelectromagnetics 32, 7 (2011)

4. C.F. Williams, G.M. Geroni, A. Pirog, J. Lees, Applied Physics Letters, 109, 9 (2016)

5. A.R. Bahtizin, V.Y. Bortalevich, E.L. Loginov, A.I. Soldatov, Journal of Physics: Conference Series (2019)

6. S.I. Bortalevich, V.U. Chinaliev, E.L. Loginov, A.I. Soldatov, Journal of Physics: Conference Series (2019)

7. V.N. Selivanov, M.B. Barannik, V.A. Bilin, et al., Vestnik MGTU, 1 (2018)

8. E.P. Grabchak, V.V. Grigoriev, E.L. Loginov, Lecture Notes in Electrical Engineering (2020)

9. E.P. Grabchak, E.L. Loginov, V.V. Grigoriev, Lecture Notes in Electrical Engineering, 729 (2021)

10. E.L. Loginov, V.V. Grigoriev, V.S. Balandin, A.A. Shkuta, P.A. Boyko, IOP Conference Series: Materials Science and Engineering, IIET 2020 (2020)

11. E.L. Loginov, V.V. Grigoriev, A.A. Shkuta, V.Y. Bortalevich, D.D. Sorokin, IOP Conference Series: Materials Science and Engineering (2019)

12. E.L. Loginov, V.V. Grigoriev, A.A. Shkuta, D.D. Sorokin, V.Y. Bortalevich, IOP Conference Series: Materials Science and Engineering (2019)

13. V.V. Grigor'ev, L.G. Dumbadze, V.Yu. Leonov, Journal of Computer and Systems Sciences International this link is disabled, 46, 6 (2007)

14. E.P. Grabchak, E.L. Loginov, Security Problems (Security - 2020): Proceedings of the II International Scientific and Practical Conference (Ufa State Aviation Technical University, Ufa, 2020)

15. E.P. Grabchak, V.V. Grigoriev, E.L. Loginov, New information technologies and systems. Collection of scientific articles based on the materials of the XVII International Scientific and Technical Conference (Penza State University, Penza, 2020) 